Article

\title{
Textile Design and Product Innovations from Adinkra and Bo- golanfini Ideographic Mergers
}

\author{
Joseph Ampadu ${ }^{1,}$, Richard Acquaye ${ }^{2}$, , Genevieve Adjei Appoh ${ }^{3}$ \\ ${ }^{1}$ Department of Fashion Design and Textile Studies, Cape Coast Technical University, Cape Coast, Ghana \\ ${ }^{2}$ Department of Textile Design and Technology, Takoradi Technical University, Takoradi, Ghana \\ ${ }^{3}$ Department of Fashion Design and Technology, Takoradi Technical University, Takoradi, Ghana \\ *Correspondence: joseph.ampadu@cctu.edu.gh
}

How to cite this paper: Ampadu, J., Acquaye, R., \& Adjei Appoh, G. (2022). Textile Design and Product Innovations from Adinkra and Bogolanfini Ideographic Mergers. Journal of Art and Design, 1(1), 28-45. Retrieved from https://www.scipublications.com/journal/index.php/jad/article/view/231

Received: December 29, 2021 Accepted: February 23, 2022 Published: February 24, 2022

Copyright: (C) 2022 by the authors. Submitted for possible open access publication under the terms and conditions of the Creative Commons Attribution (CC BY) license (http://creativecommons.org/licenses/by/4.0/).

\begin{abstract}
This study seeks to introduce an interactive design concept by merging Adinkra and Bogolanfini symbols into textile designs and convert them into utilitarian products. The qualitative research design method and the human-centred design approach were employed to identify, understand and assess how effective Adinkra and Bogolanfini ideographs can be adapted for textile designs and other product applications. The target samples for this study comprises variety of Adinkra symbols and Bogolanfini patterns. The significance of sampling in this study was to select suitable kinds of Adinkra and Bogolanfini patterns. Lines, shapes and texture were utilised to determine which particular symbols were included and which were not suitable. The CorelDraw vector software was used to convert and develop images of the Adinkra symbols and Bògòlanfini patterns and then manipulated into the final textile design. The design outcomes are indicative of the fact that varieties of Adinkra symbols and Bogolanfini patterns are prospective image resources for textile designs. The study recommends that textile design students and practitioners at various institutions and dispositions should be encouraged to explore the breadth of ideographs available across the West African sub-region for design ideas.
\end{abstract}

Keywords: Textiles; Symbols; Textile Design; Adinkra; Bogolanfini

\section{Introduction}

This study seeks to introduce an interactive design concept by merging Adinkra and Bogolanfini symbols into textile surface designs and convert them into a variety of products. Textile designing encompasses the creating of designs and structures for knitted, woven, nonwoven, or embellishment of fabrics. It involves a creative field that includes fashion design, carpet manufacturing and any other textile fabrics and clothing-related endeavour. Textile design fulfils a variety of purposes in our lives, for example, clothing, carpets, drapes, towels and rugs are all products of textile design [1]. Design is considered by many as key to innovation which is acknowledged as crucial for change. Textile design mergers, evolution and innovations has been an essential part of human development and culture. Even though, social change has influenced this phenomenon to some extent, however, commercial implications have been a major driver in textile design development over time [2].

Developing textile design and production concepts according to [3] is bringing together historical references, technological innovations, production techniques and process. As a result, designers and design researchers rarely work alone but are required to work collaboratively across disciplines and to communicate with technologists and manufacturers. Working in this way enables new ideas to be tested towards implementation within the relevant context, facilitating innovations for a long period of time. This in turn would lead to several design variations and product outcomes in textile design practice. 
The Adinkra symbol of the Ashanti of Ghana and the Bogolanfini of Mali are design themes or motifs that can serve as a source of textile design adaptation. Adinkra symbols are described as visual, symbolic pictures or motives that represent concept or aphorisms. Adinkra symbols are used extensively to decorate colourful patterned cloth by textile designers and pottery among the Asantes of Asante Kingdom. In recent times, designers adopt Adinkra symbols in creating and decorating other accessories than cloth [4]. They are also incorporated into walls and other architectural features. Adinkra fabrics are often made by woodcut sign writing as well as screen printing. Adinkra symbols appear on some traditional Akan goldweights. The symbols are also carved on stools for domestic and ritual use [5].

Bogolanfini, on the other hand, represents expressive culture for the people of Mali. The Bogolanfini patterns communicate many tribal stories and refer to animals, historical events, religion and mythologies of the tribe. The meaning of the symbols can differentiate between regions, tribes and where the cloth was created, but there are some common understandings of what Bogolanfini symbols are and what they represents. Originally, women are wrapped in a traditional piece of Bogolanfini once they are initiated into adulthood. It is believed that the patterns within the cloth are imbued with Nyama, which is a vital life force, energy or strength [6].

It is evident that separately, the two design ideographs have been important sources of design creation for generations. It is anticipated that Adinkra and Bogolanfini symbol combinations in designing will give a great output of endless textile design permutation. This study is meant to introduce the concept of textile design adaptation from selected Adinkra symbols and Bogolanfini patterns. It will, therefore, serve as a source of design inspiration for textile students and designers in bringing variety in their textile designing and also expose new areas or other avenues for textile designers to explore. This study, therefore, experiment with a combination of Ghanaian Adinkra symbols and Malian Bogolanfini symbols to create technically advantageous and commercially relevant surface patterning opportunities for textile fabrics.

\subsection{Visual Analysis: Adinkra Symbols}

Adinkra symbols according to [4] are visual representation of concepts and aphorism developed by the Akan people of Ghana. Adinkra symbols are comprehensively used in pottery, advertising, fabrics, and logos. They can also be found on traditional Akan gold weights, architectural buildings, sculptures along with stools used for traditional rituals. Apart from the symbols being a decorative objects or drawings, it also conveys ancient traditional wisdom relevant to aspects of life or the environment. Most of the Adinkra symbols have meanings associated to proverbs, such as the Sankofa symbol. Sankofa, in the Twi language, translates in English to" reach back and get it" (san - to return; ko to go; fa - to look, to seek and take) or the Adinkra symbol of a bird with its head turned backwards taking an egg off its back, or of a stylised heart shape. Figure 1 is a selection of Adinkra symbols. 


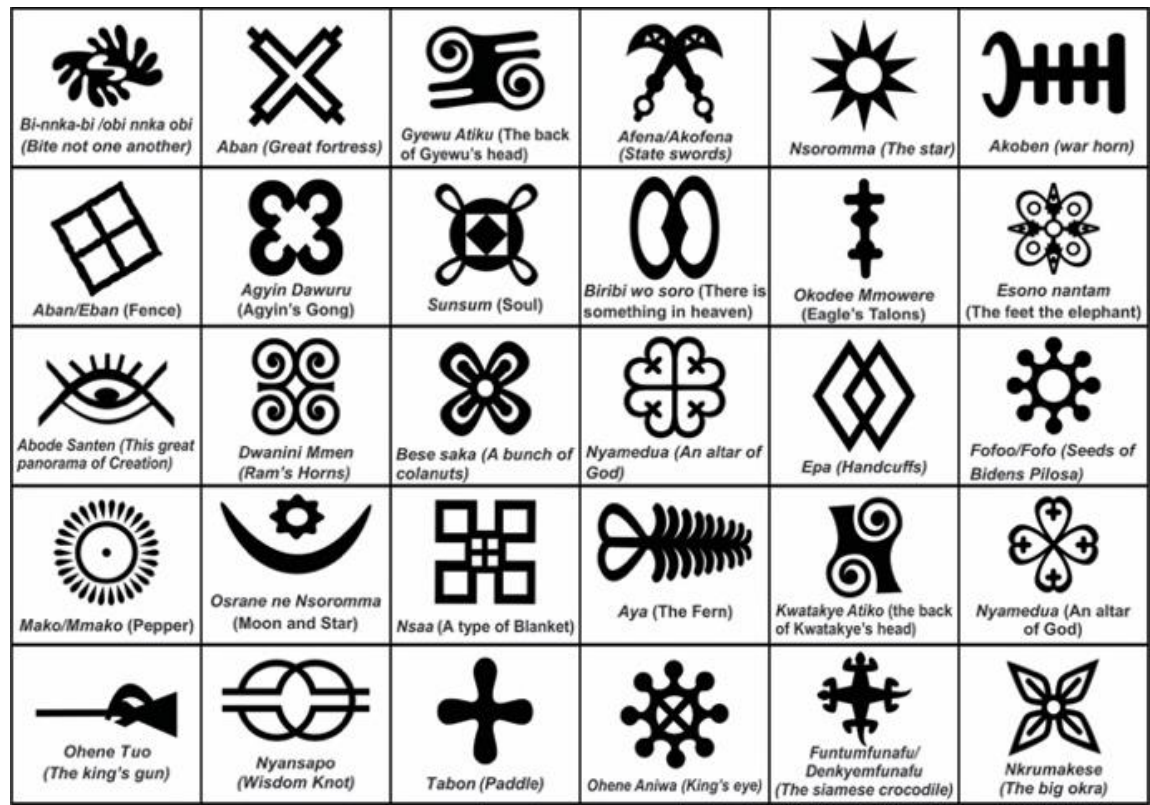

Figure 1. Adinkra symbols. Source: [10]

\subsection{Visual Analysis Bogolanfini}

Bogolanfini according to [7], is commonly known as "mud cloth," a painted handwoven cloth, made in Mali. Dyes are made from mud and leave to create traditional white patterns on black backgrounds. Luke-Boone, [8] explained the term mudcloth as the literal translation of Bogolanfini, which is a cloth decorated by women in the Bamana-speaking region of Mali. In Bamana language, bogo means "mud," and lan means "traces of." The most common Bogolanfini cloth has white geometric designs against a black background. Polakoff [9] asserts that among the most elusive and difficult to classify of all the fabrics in Africa - both in a technical as well as a design sense - has been the mud cloth of the Bambara (Bamana) peoples of Mali. Mud cloth is variously referred to as Bogolanfini cloth in Mali, both words mean that the designs are made with mud. Polakoff [9] further posit that, Bogolanfini is thought to have originated in Beledougou, spreading eventually through other parts of the west-central area of Mali. It is in Beledougou that the finest design work is done.

Bògòlanfini according to [6], is a skill practised precisely to the country of Mali. It involves the dying of handmade Malian clothing and fabrics. The dyes used are made from mud which is rich in iron to create black colours and plants that produce a range of colours. Bògòlanfini symbolises expressive culture for the people of Mali. The patterns dyed on the fabric communicate a lot of tribal stories and refer to animals, historical events, religion, and mythologies of the tribe. The cloth is majorly dyed by females who are fluent in this iconographic language. The meaning of the symbols can differentiate between regions, tribes and where the cloth was created, but there are some collective understandings of what Bògòlanfini is and what it signifies. Once women are initiated into adulthood, they are wrapped in traditional piece of Bògòlanfini. It is understood that the patterns within the cloth are infused with Nyama, which is a vigorous life force, energy or strength.

Male hunters wear Bògòlanfini, who are also granted with Nyama to protect them during their hunts. The hunter's mud-cloth is dyed a rusted colour to camouflage them as well as represent blood for their hunted animals. Black backgrounds with white symbols are common colour combinations that are used for storytelling. Other common representations illustrated in mud-cloth are major events such as the birth, marriage or death of someone or even an individual's social status, or occupation [6]. In making mudcloth, the traditional way according to [8], the artist embellishes the cloth with geometric shapes 
that are often abstract renditions of everyday objects such as cowry shells. Using bamboo and other flat tools, an artist outlines patterns she wishes to create and areas to be dyed with the fermented mud dye over the entire of cloth. Then she fills in the patterns throughout the entire length of the fabric and then allow to dry. The fabric is later washed to reveal the amazing patterns; the fabric is then finished.

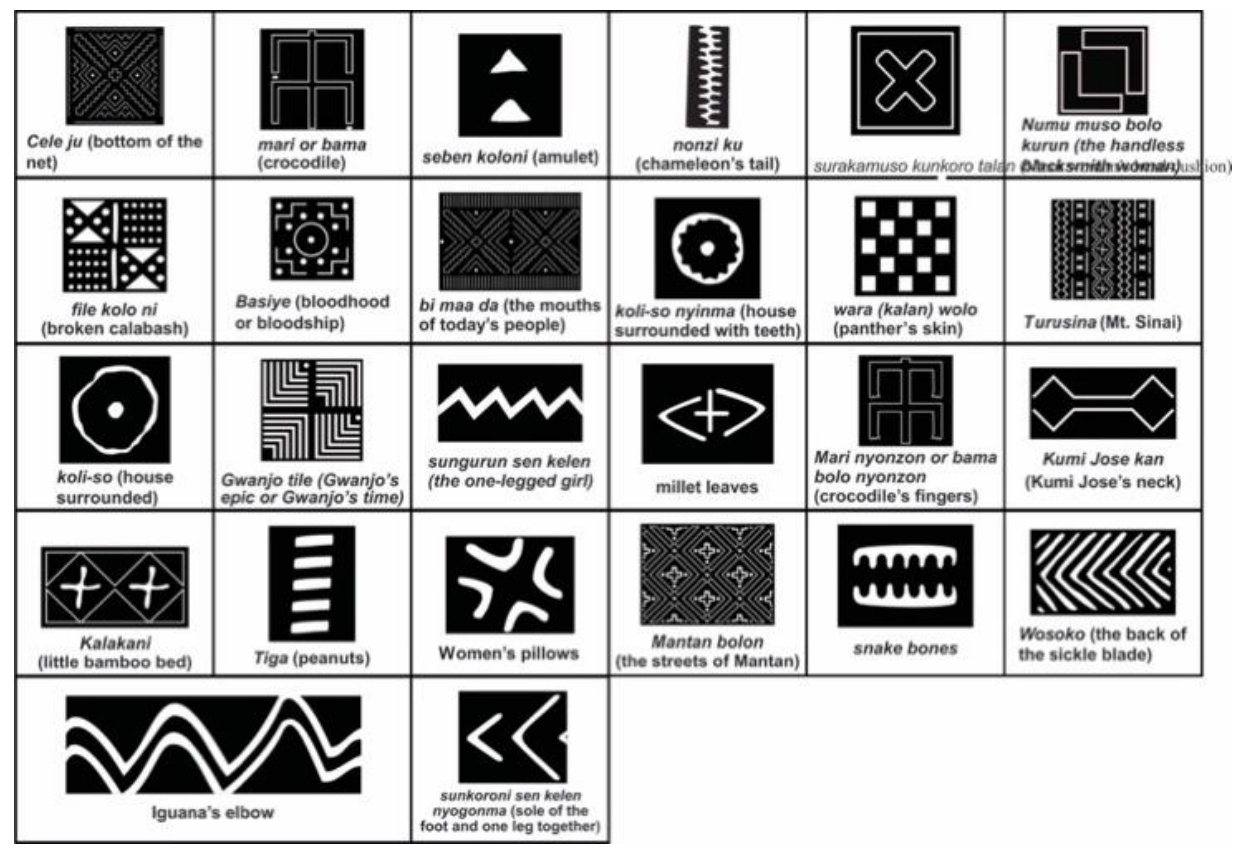

Figure 2. Bogolanfini patterns. Source: [11]

\section{Materials and Methods}

\subsection{Generation of the Textile Designs}

This segment outlines the steps used in generating the textile designs from selected Adinkra symbols and Bògòlanfini patterns. The conversion and generation of the Adinkra symbols and Bògòlanfini patterns to the final design was done in stages using CorelDraw X7. The steps followed to generate the various designs from the Adinkra symbols and Bògòlanfini patterns to the final designs are outlined in the steps listed below:

- Opening the image in CorelDraw

- $\quad$ Selecting the image on CorelDraw

- The images were thereafter sketched and develop to get motifs for the design.

- $\quad$ Adjusting the size and image quality

- Converting the image into outlines and application of other features by using the Bezier tool.

- $\quad$ Resizing, mirror reflecting, arranging and defining the patterns.

- Colour application and other background effects.

\subsection{Design Project - One}

Basiye (bloodhood or bloodship) - Bogolanfini pattern and Okodee Mmowere (Eagle's Talons) - Adinkra symbol) 


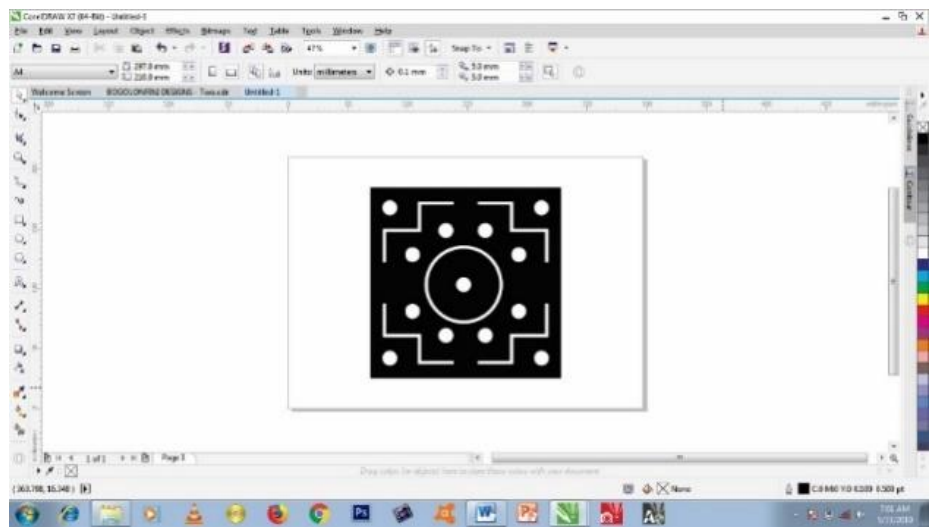

Figure 3. Basiye (Bloodhood or bloodship) opened in CorelDraw

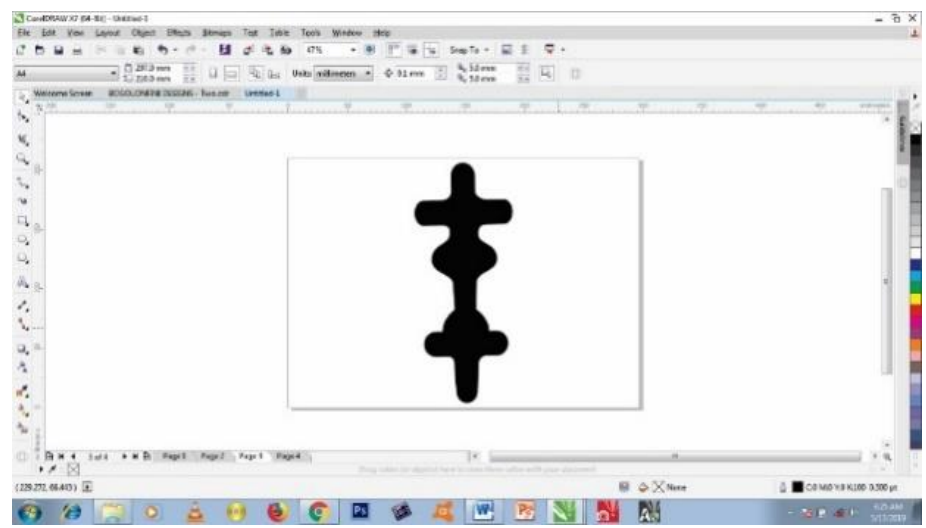

Figure 4. Okodee Mmowere (Eagle's Talons) opened in CorelDraw

The images of the Bogolanfini pattern and Adinkra symbol were opened in CorelDraw as shown in Figure 3 and Figure 4 respectively. The symbols were converted to a vector objects with the help of the trace bitmap command. The Bogolanfini pattern was repeated two times vertically and horizontally respectively. The Adinkra symbol was place in between the Bogolanfini pattern. Both images were coloured, grouped and intersected with the aid of the rectangle tool to obtain a unit repeat of the design. The unit repeat of the design was repeated vertically and horizontally. Colour was applied to the background to obtain the design in Figure 5 and Figure 6.

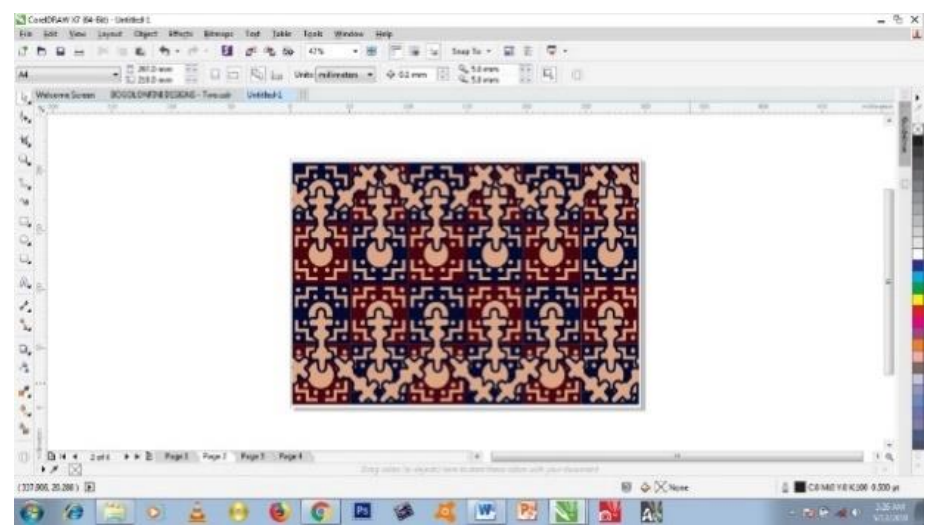

Figure 5. Final design as represented in CorelDraw 


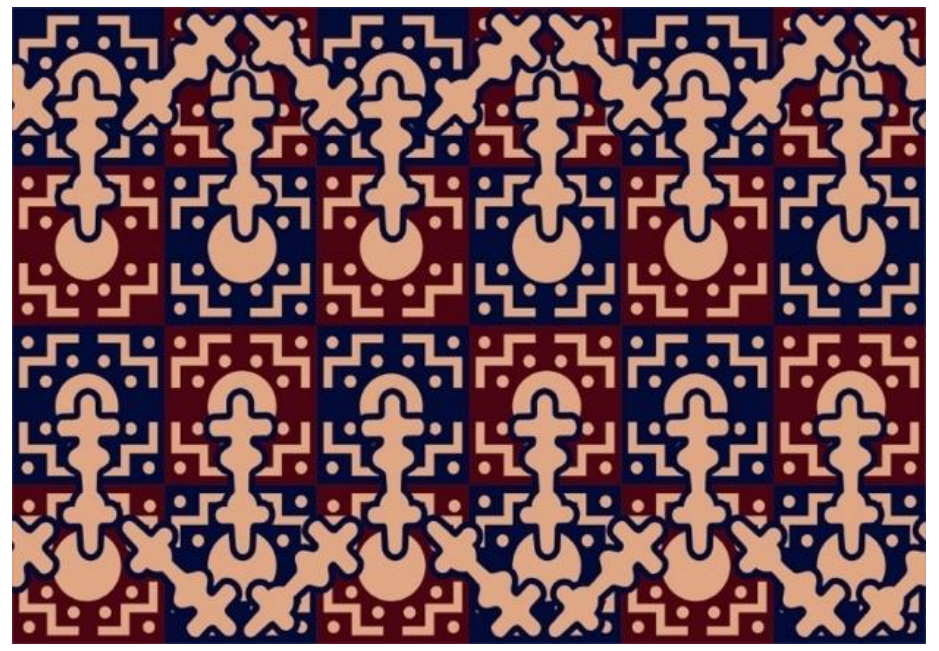

Figure 6. Final design

\subsection{Design Project - Two}

Wara (kalan) wolo (panther's skin) - Bogolanfini pattern and Mako/Mmako (Pepper) Adinkra symbol

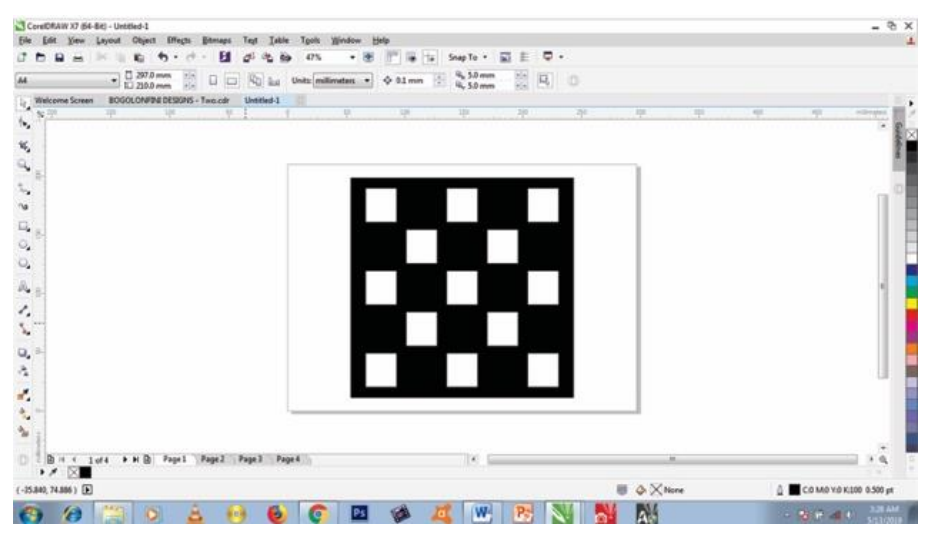

Figure 7. Wara (kalan) wolo (panther's skin) opened in CorelDraw

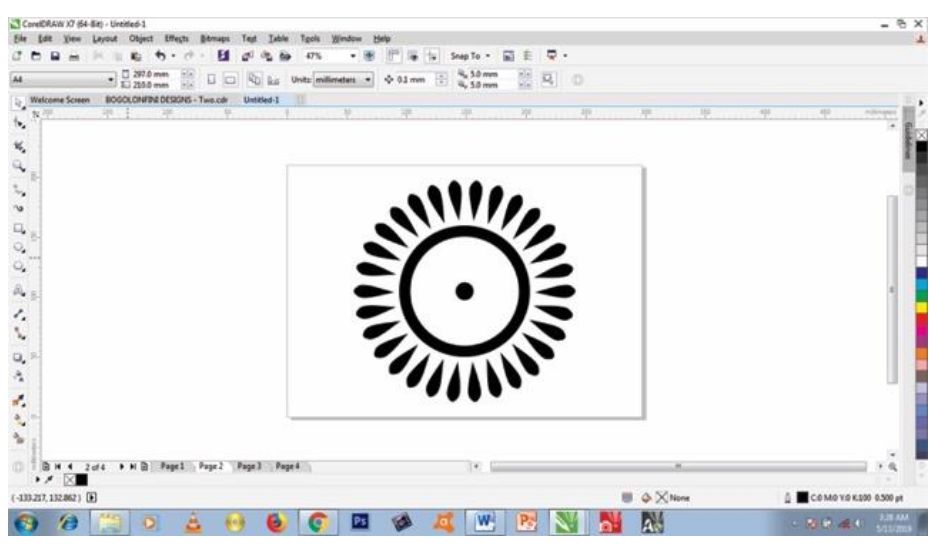

Figure 8. Mako/Mmako (Pepper) opened in CorelDraw

The images of the Bogolanfini pattern and Adinkra symbol were opened in CorelDraw as shown in Figure 7 and Figure 8 respectively. The images were converted to a vector object with the help of the trace bitmap command. The Bogolanfini pattern was divided into two counter changed respectively. The Adinkra symbol was coloured and 
placed on top of the Bogolanfini pattern. Both images were selected and repeated four times with the second and fourth repeats being mirrored vertically. The four repeats were selected, grouped and repeated twice vertically. Colour was applied to the background to obtain the design in Figure 9 and Figure 100.

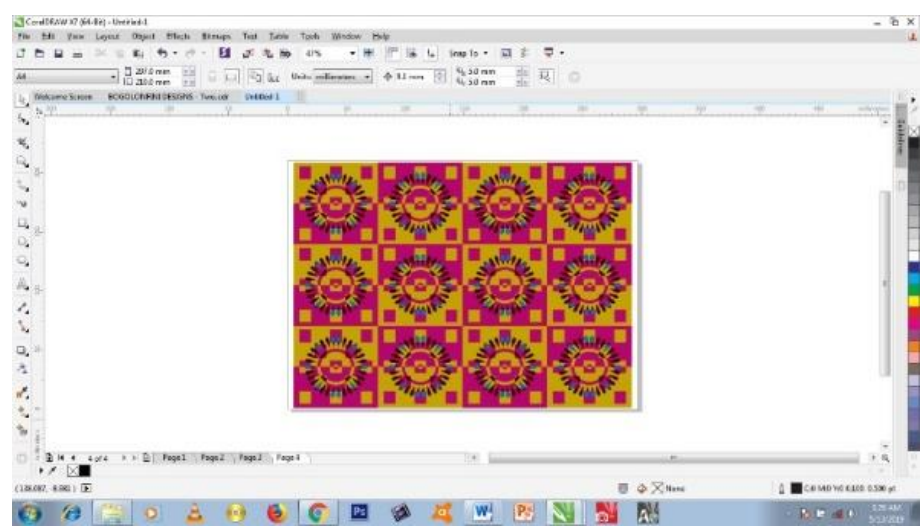

Figure 9. Final design as represented in CorelDraw

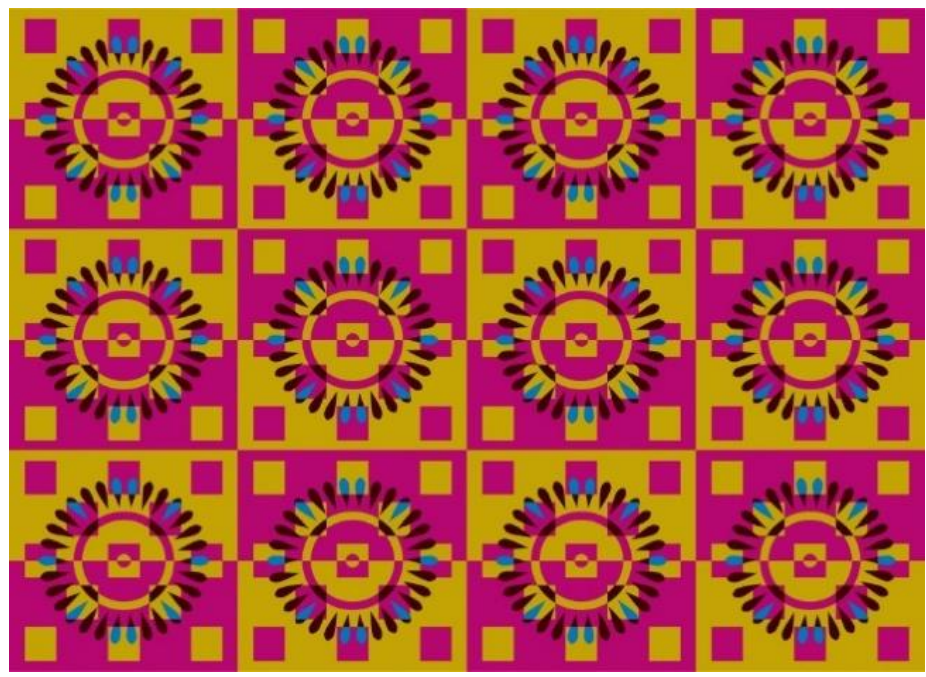

Figure 10. Final Design

\subsection{Design Project - Three}

Juguru Fara (The feet of the tortoise) - Bogolanfini pattern and Ohene (King) - Adinkra symbol

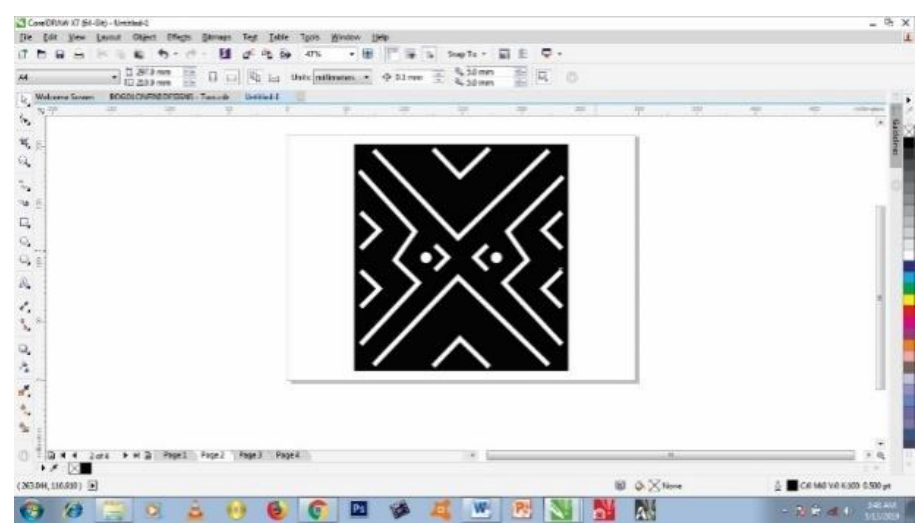

Figure 11. Juguru Fara (The feet of the tortoise) opened in CorelDraw 


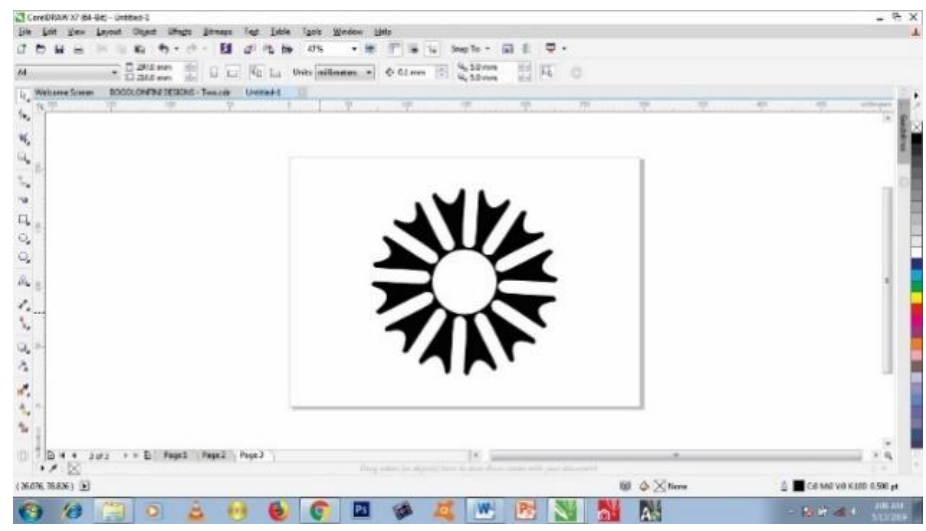

Figure 12. Ohene (King) opened in CorelDraw

The images of the Bogolanfini pattern and Adinkra symbol were opened in CorelDraw as shown in Figure 111 and Figure 122 respectively. The images were converted to a vector object with the help of the trace bitmap command. The Bogolanfini pattern was given colours and repeated vertically and horizontally to serve as textures for the design. A series of concentric shapes was applied out of the Adinkra symbol. The Adinkra symbol was then coloured repeated severally on the Bogolanfini pattern. Colour was applied to the background to obtain the design in Figure 133 and Figure 144.

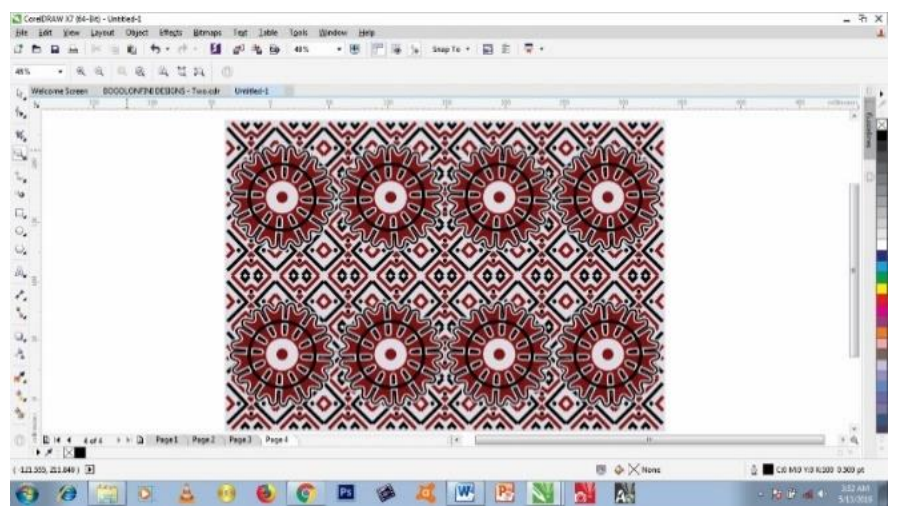

Figure 13. Final design as represented in CorelDraw

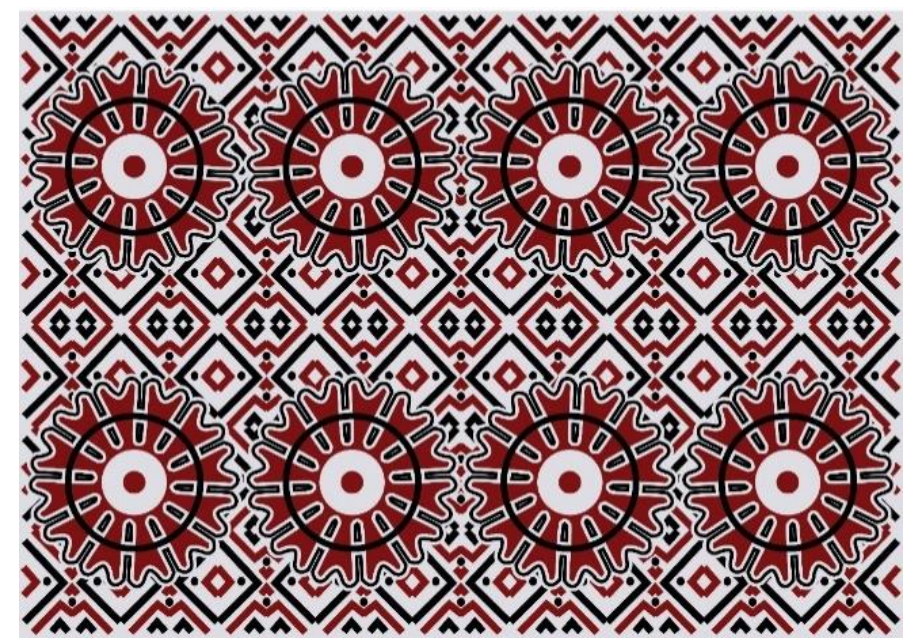

Figure 14. Final Design 


\subsection{Design Project - Four}

Surakamuso kunkoro talan (Moor woman's head-cushion) - Bogolanfini pattern and Bi$n n k a-b i$ lobi nnka obi (Bite not one another) - Adinkra symbol

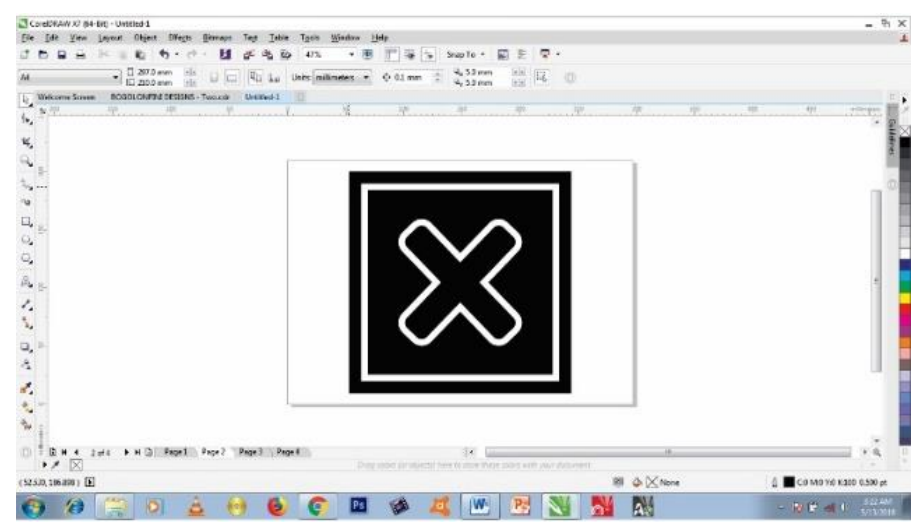

Figure 15. Surakamuso kunkoro talan (Moor woman's head-cushion) opened in CorelDraw

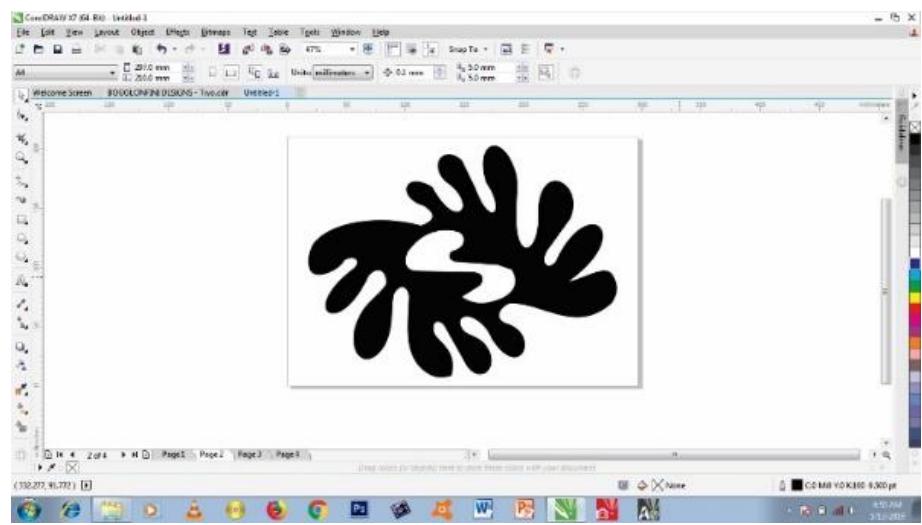

Figure 16. Bi-nnka-bi lobi nnka obi (Bite not one another) opened in CorelDraw

The images of the Bogolanfini pattern and Adinkra symbol were opened in CorelDraw as shown in Figure 155 and Figure 166 respectively. The images were converted to a vector object with the help of the trace bitmap command. The Adinkra symbol was repeated twice before it was rotated at 180 degrees. The rotated symbol was repeated down and mirrored vertically. The Bogolanfini pattern was placed opposite each other at $90^{\circ}$ degrees and $180^{\circ}$ respectively in between the Adinkra symbol and trimmed to get a half drop arrangement. The patterns were then repeated severally to fill the document page. Colours were applied to the patterns and background to obtain the design in Figure 177 and Figure 188.

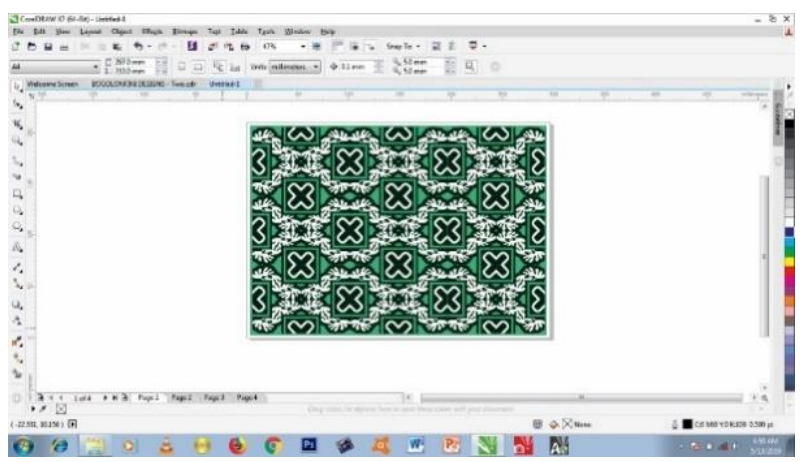

Figure 17. Final design as represented in CorelDraw 


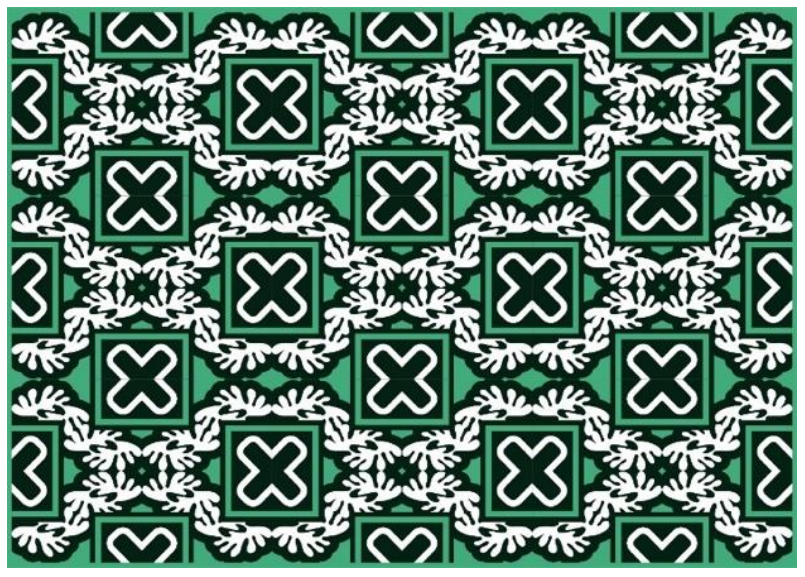

Figure 18. Final design

\subsection{Design Project - Five}

Nonzi ku (chameleon's tail) - Bogolanfini pattern and Okodee Mmowere (Eagle's Talons) - Adinkra symbol.

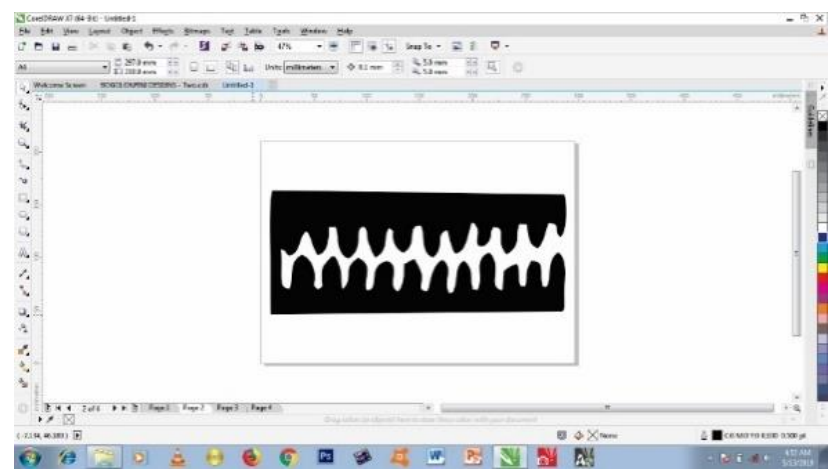

Figure 19. Nonzi ku (chameleon's tail) opened in CorelDraw

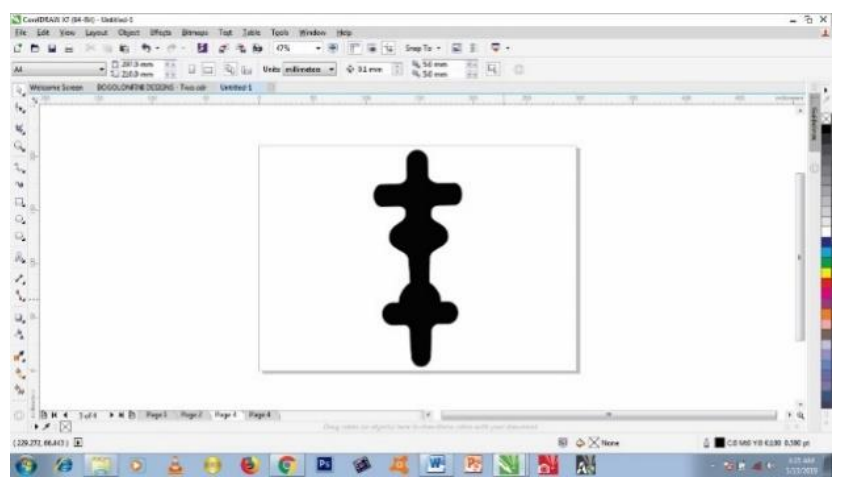

Figure 20. Okodee Mmowere (Eagle's Talons) opened in CorelDraw

The images of the Bogolanfini pattern and Adinkra symbol were opened in CorelDraw as shown in Figure 199 and Figure 200 respectively. The images were converted to a vector object with the help of the trace bitmap command. The Adinkra symbol was repeated vertically four times, grouped and repeated two times horizontally. The Zigzag design in the Bogolanfini pattern was repeated and placed in between the Adinkra symbol. An oval symbol was drawn and placed in between both pattern to create a rhythm in the design. Colours were applied to the patterns and background to obtain the design in Figure 211 and Figure 222. 


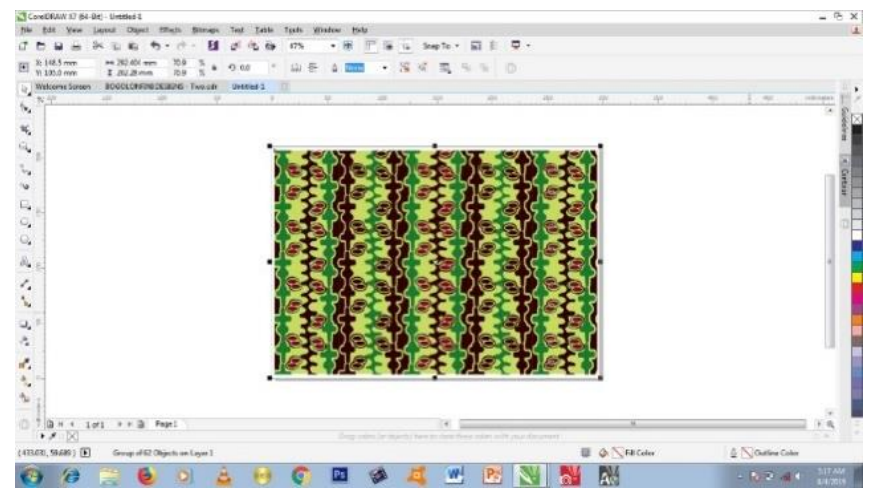

Figure 21. Final design as represented in CorelDraw

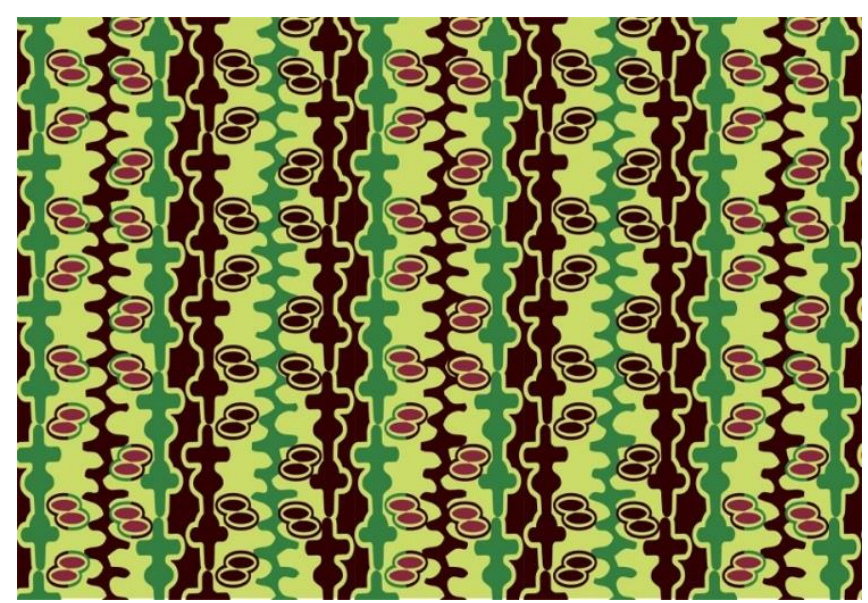

Figure 22. Final design

\subsection{Design Project - Six}

Basiye (bloodhood or bloodship) - Bogolanfini pattern and Ohene (King) - Adinkra symbol

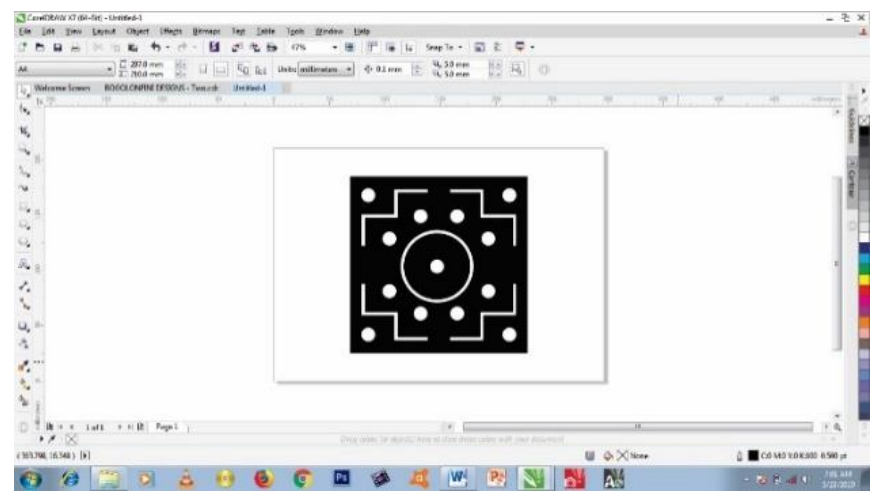

Figure 23. Basiye (bloodhood) - Bogolanfini pattern opened in CorelDraw 


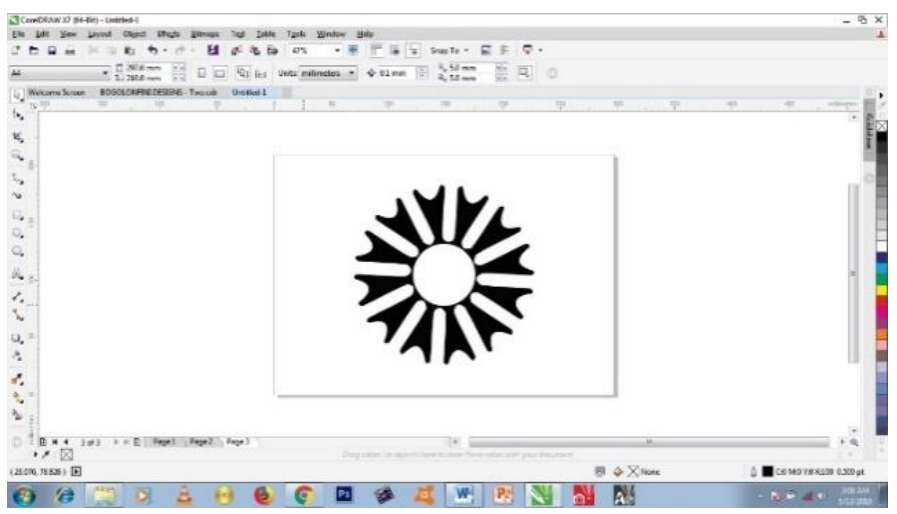

Figure 24. Ohene (King) - Adinkra symbol opened in CorelDraw

The images of the Bogolanfini pattern and Adinkra symbol were opened in CorelDraw as shown in Figure 233 and Figure 244 respectively. The images were converted to a vector object with the help of the trace bitmap command. The images were given colour and placed within lines and a scissors-like shape. The background colour was given a wax effect to with the help of the eraser tool. The images used as motifs were repeated to get the design in Figure 255 and Figure 266.

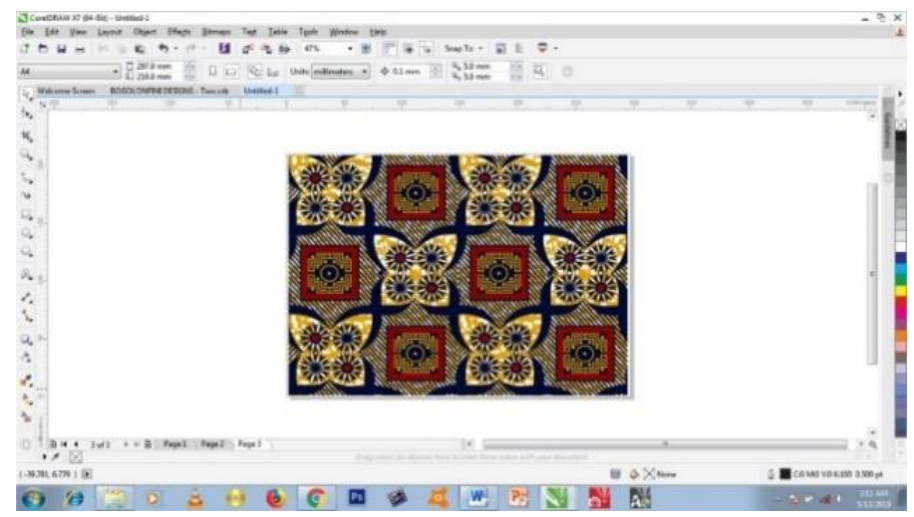

Figure 25. Final design opened in CorelDraw

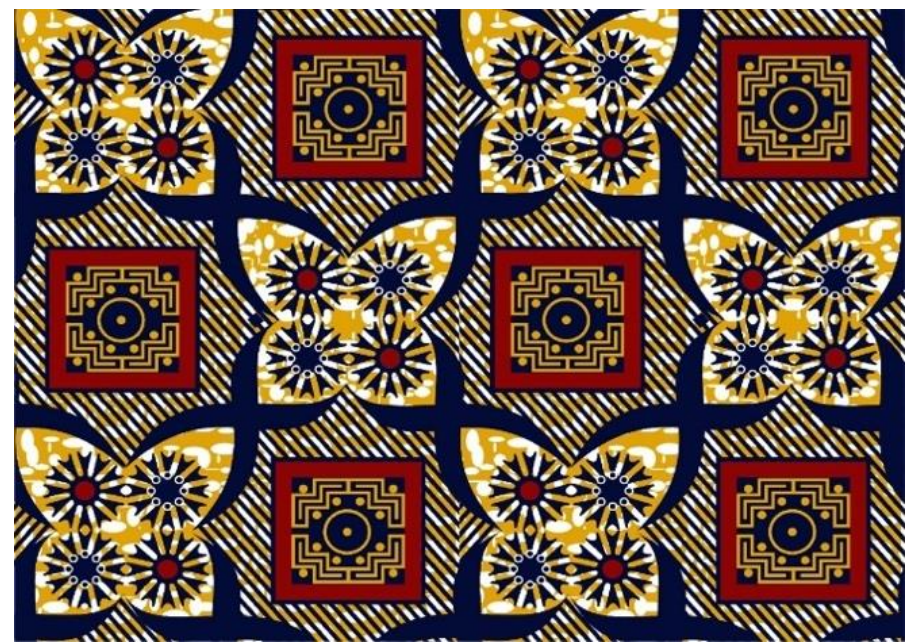

Figure 26. Final design 


\subsection{Design Project - Seven}

Seben Koloni (The small old amulet) - Bogolanfini pattern and Nsaa (A type of Blanket) - Adinkra symbol.

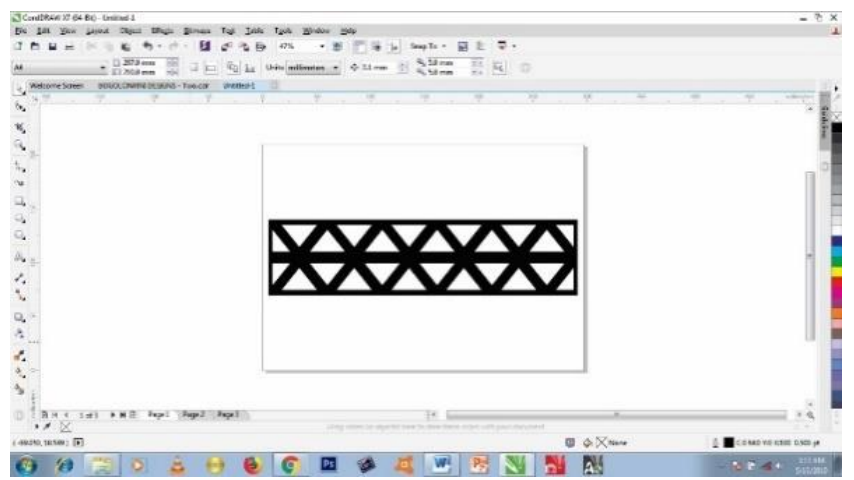

Figure 27. Seben Koloni (The small old amulet) - Bogolanfini pattern opened in CorelDraw

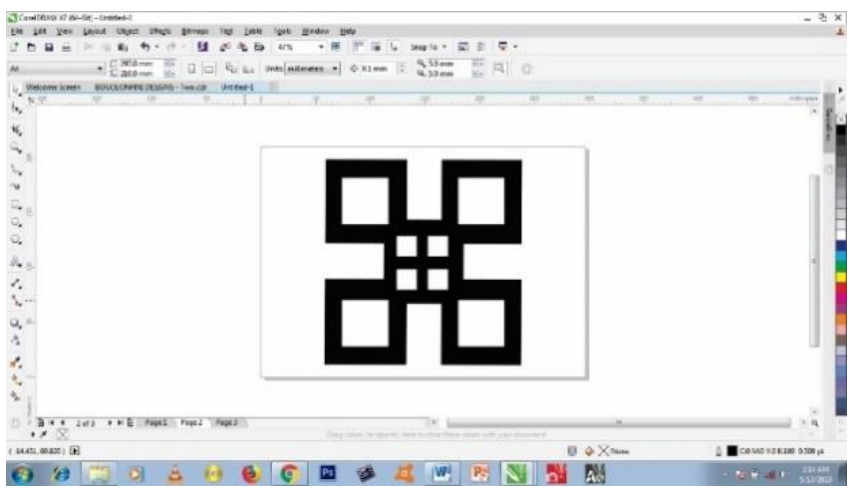

Figure 28. (A type of Blanket) - Adinkra symbol opened in CorelDraw

The images of the Bogolanfini pattern and Adinkra symbol were opened in CorelDraw as shown in Figure 277 and Figure 288 respectively. The images were converted to a vector object with the help of the trace bitmap command. The Adinkra was repeated three times and placed beside the Bogolanfini pattern. The two images were coloured, selected and repeated horizontally four times. All the images repeated horizontally were selected and repeated vertically. The images repeated vertically were then mirrored horizontally. Colour was applied to the background to obtain the design in Figure 299 and Figure 300 .

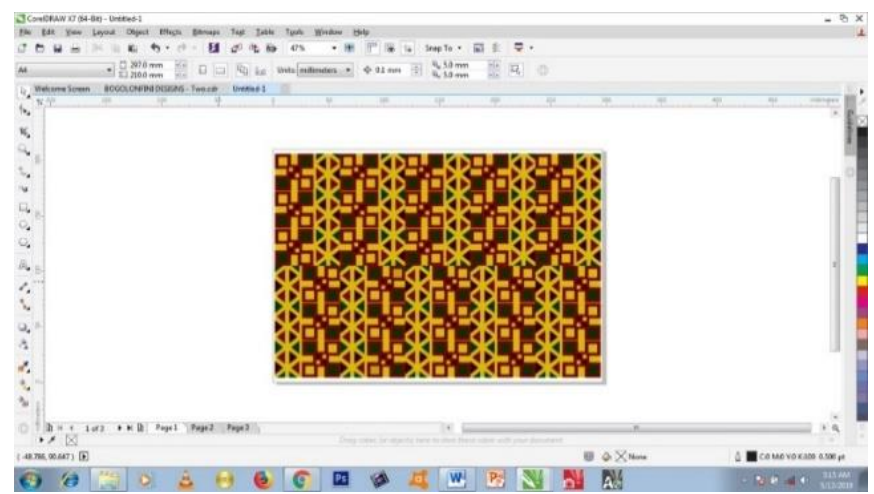

Figure 29. Final design opened in CorelDraw 


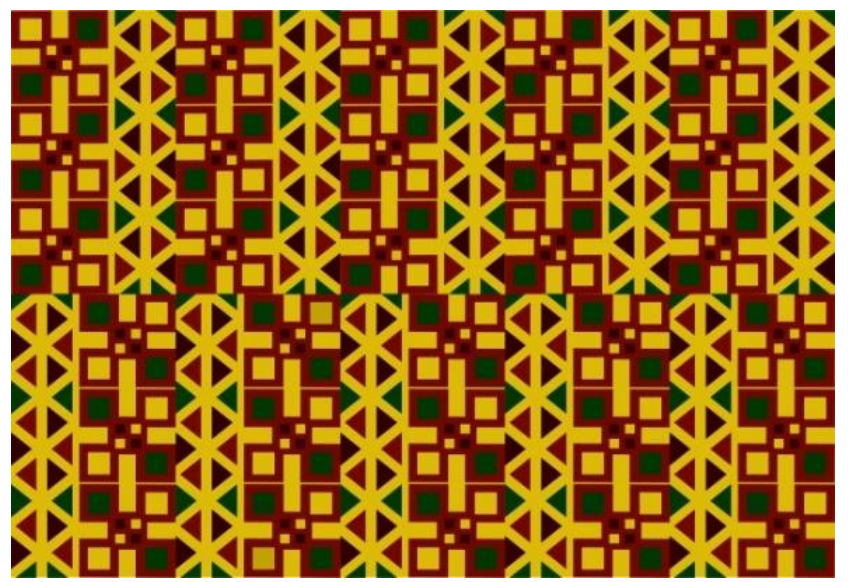

Figure 30. Final design

\subsection{Design Project - Eight}

Nonzi ku (chameleon's tail) - Bogolanfini pattern and Kwatakye Atiko (the back of Kwatakye's head) - Adinkra symbol.

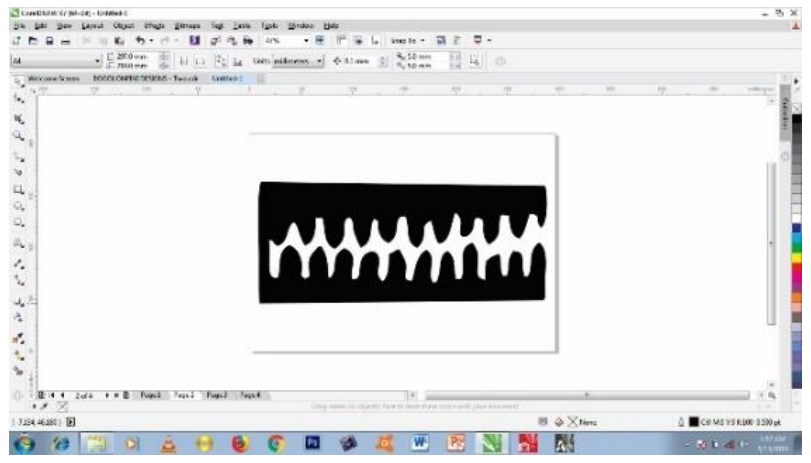

Figure 31. Nonzi ku (chameleon's tail) - Bogolanfini pattern opened in CorelDraw

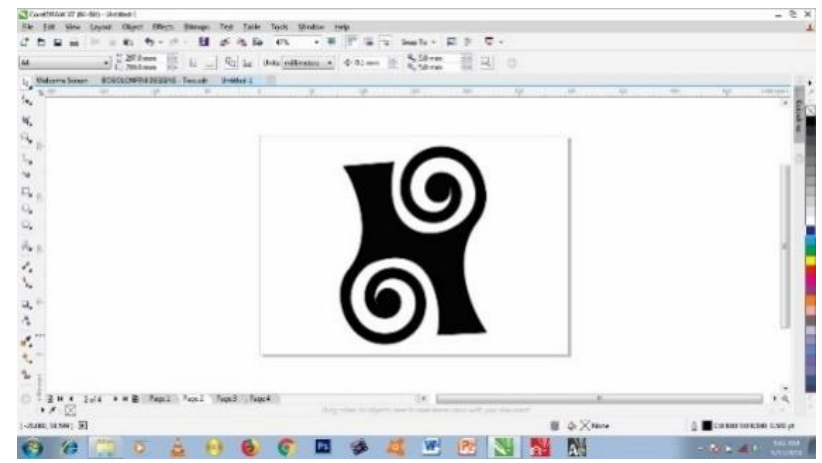

Figure 32. Kwatakye Atiko (the back of Kwatakye's head) - Adinkra symbol opened in CorelDraw

The images of the Bogolanfini pattern and Adinkra symbol were opened in CorelDraw as shown in Figure 311 and Figure 322 respectively. The images were converted to a vector object with the help of the trace bitmap command. The Adinkra symbol was repeated two times vertically and horizontally respectively. The crossed shape in the Bogolanfini pattern was place in between the Adinkra symbol. Both images were coloured, grouped and intersected with the aid of the rectangle tool to obtain a unit repeat of the design. The unit repeat of the design was repeated vertically and horizontally. Colour was applied to the background to obtain the design in Figure 333 and Figure 344. 


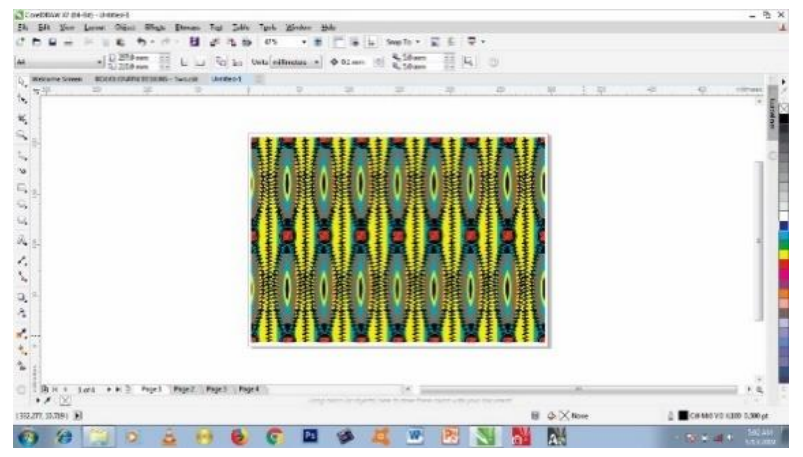

Figure 33. Final design opened in CorelDraw

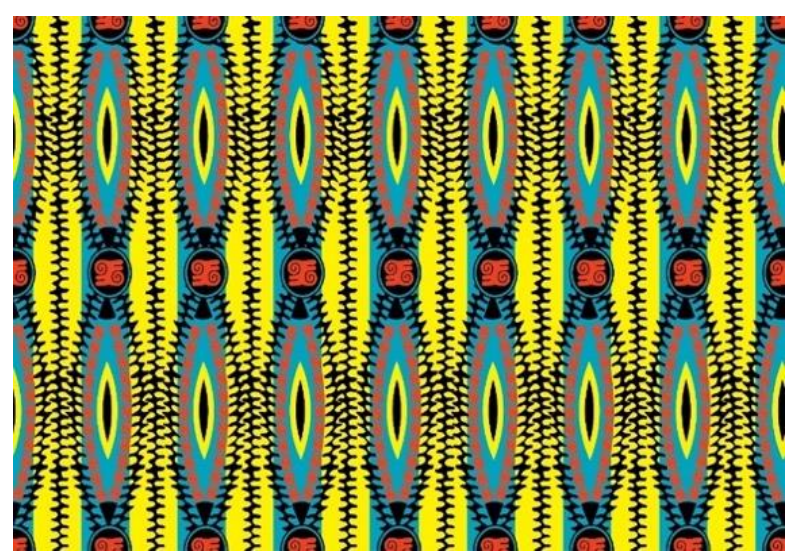

Figure 34. Final design

\subsection{Product Outcomes}

Men and Ladies Apparel

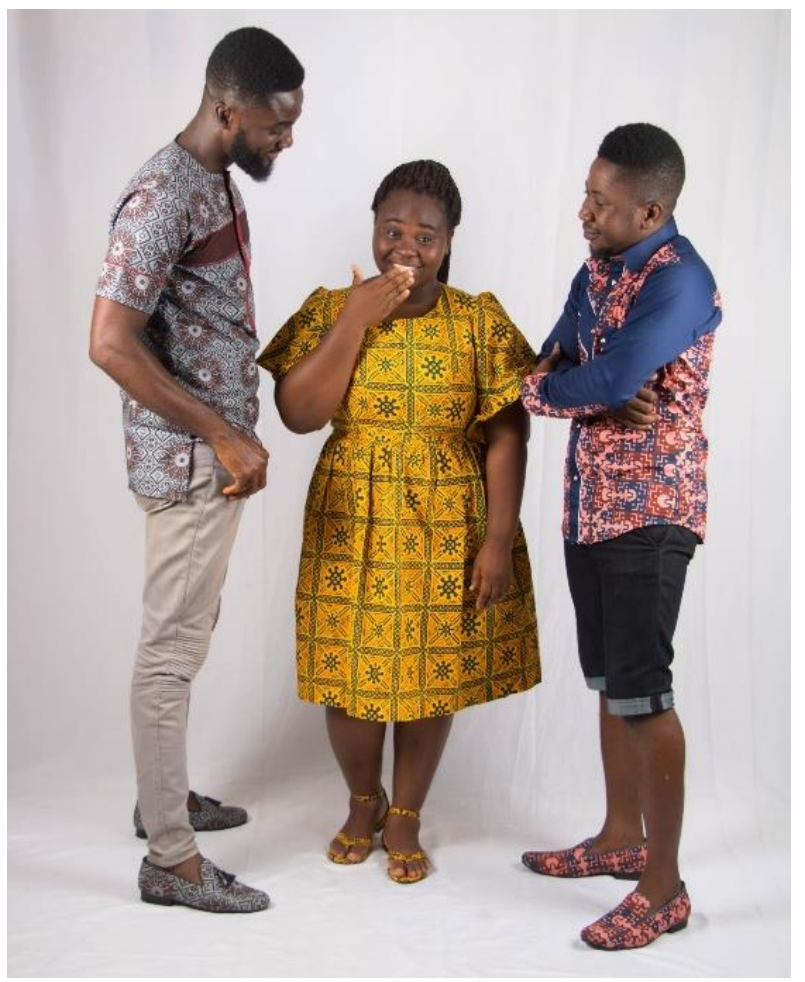

Figure 35. Men and Ladies apparel (Outsourced to Nii Addo's Design, 2020) 
Men and Ladies Footwears

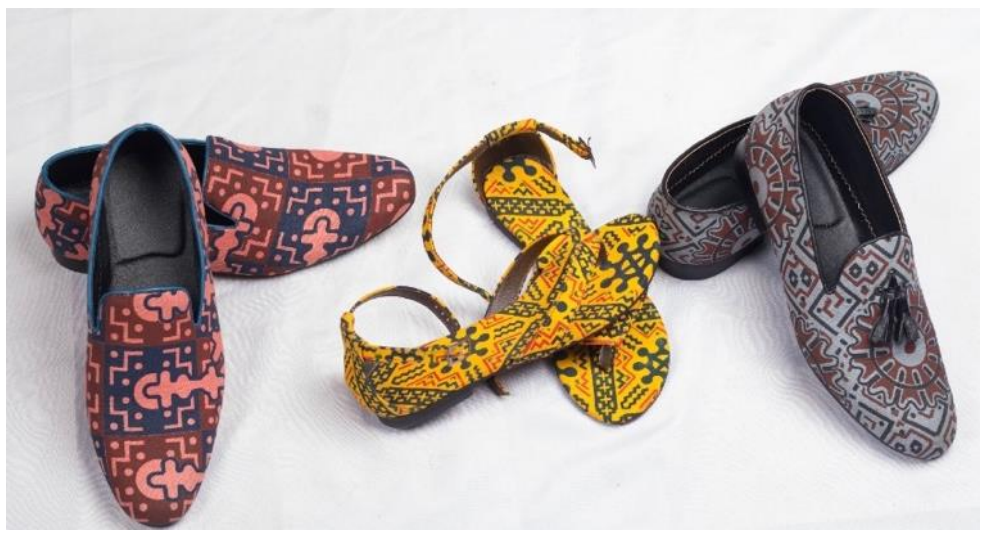

Figure 36. Men and ladies' footwear (Outsourced to Edisef Enterprise, 2020)

\section{Armrest}

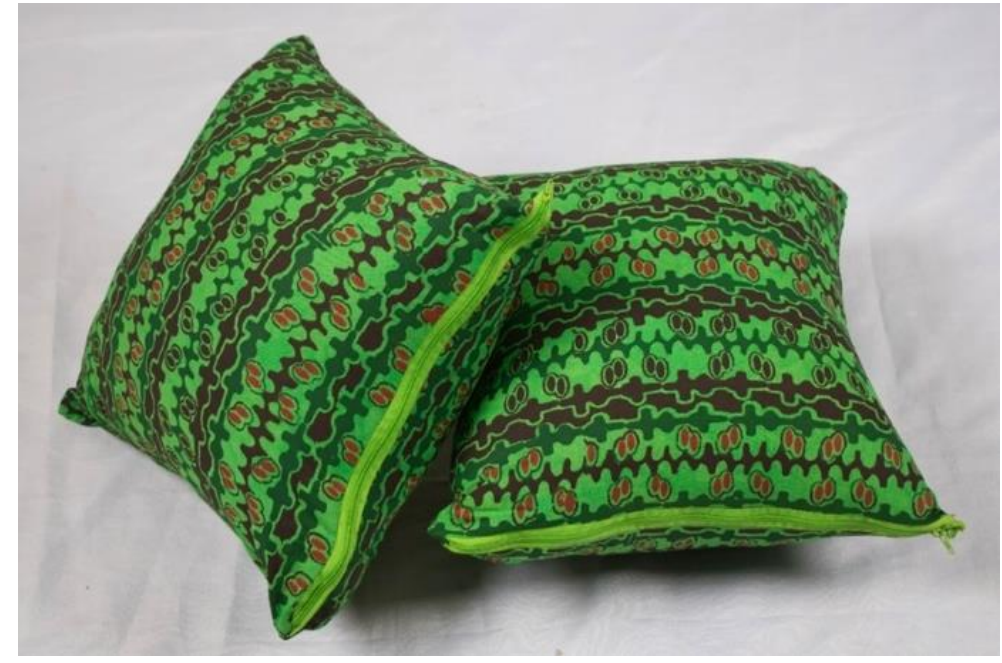

Figure 37. Armrest (Outsourced to Nii Addo's Design, 2020)

\section{Bag Pack and Accessory}

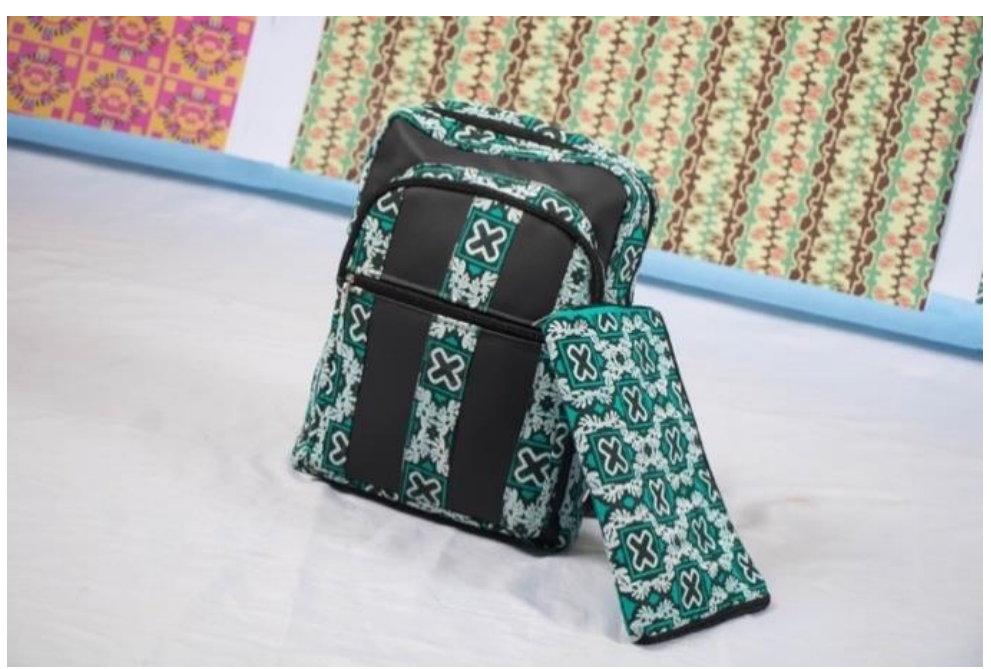

Figure 38. Bag pack and accessory (Outsourced to Galaxy Textile Collection, 2020) 


\section{Wall Hanging}

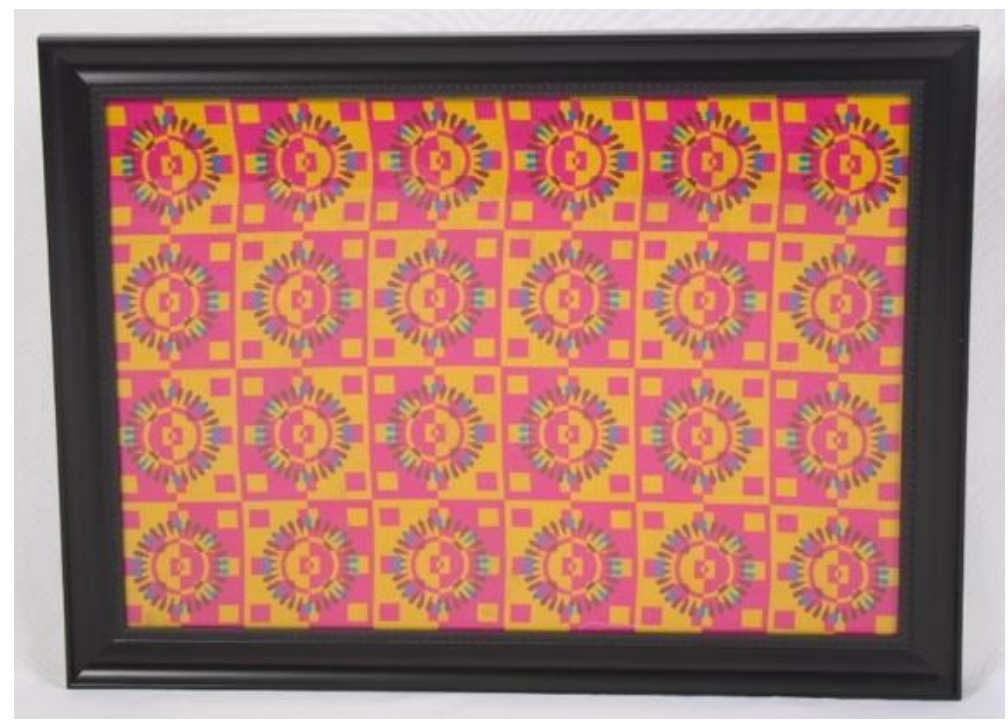

Figure 39. Wall hanging (Outsourced to Dignity Frames, 2020)

\section{Findings and Discussion}

The main objective of this study was to introduce an interesting concept in designing by merging Adinkra and Bogolanfini into textile surface designs and convert them into other products such as apparel, men and ladies' footwear, school bag, armrest and picture frame. The Adinkra symbol of the Ashantis of Ghana and the Bogolanfini of Mali are design themes or motifs that can serve as a source of textile design adaptation. Adinkra symbols described as visual, symbolic pictures or motives that represent concept or aphorisms are used extensively to decorate colourful patterned cloth by textile designers and pottery among the Ashanti's of Ashanti Kingdom. Designers in modern times use Adinkra symbols in creating and decorating other accessories than cloth. Bogolanfini, on the other hand, represents expressive culture for the people of Mali. The Bogolanfini patterns communicate many tribal stories and refer to animals, historical events, religion, and mythologies of the tribe. The meaning of the symbols can differentiate between regions, tribes and where the cloth was created, but there are some common understandings of what Bogolanfini is and what it represents.

Through the observational guide and secondary data, several Adinkra and Bogolanfini symbols were documented out of which some were selected randomly for the textile designs. The conversion and developing of images of the Adinkra symbols and Bogolanfini patterns to the final textile design was done in stages using CorelDraw. Out of about sixteen textile designs, five were selected and produced using the screen-printing technique. The textile designs produced were subsequently converted into various products such as men and ladies' apparel, men and ladies foot wears, arm rest, student bag and last but not the least wall picture frame. The results show that based on the unique characteristics of the finished printed works and products, Adinkra symbol of the Ashanti's of Ghana and the Bogolanfini of Mali can both serve as design themes or motifs for a variety of design adaptations.

\section{Conclusion}

Textile design fulfils a variety of purposes in our lives and should, therefore, not be over looked. For example, clothing, carpets, drapes, towels, and rugs are all a result of textile design. Many ideographs which represent expressive culture for most ethnic groups in Africa can be adapted and use to design textiles. These ideographs express the 
culture and various themes that relate to the history, beliefs and philosophy as well as representing objects that encapsulate evocative messages that convey traditional wisdom and aspects of life of ethnic groups in Africa.

Local textile designers and textile students can turn attention to these ideographs as design sources, and with the aid of relevant computer aided software such as the CorelDraw and Adobe Photoshop, one can come out with creative and interesting textile designs. These textile designs generated from these ideographs can be printed on textile substrate in mass production which can subsequently be converted to variety of products such as bags, apparel, curtains, footwear, etc. It could be inferred that with the proper use of the design elements based on design principles, an array of designs can be produced by sourcing themes from ideographs of ethnic groups in Africa.

Author Contributions: “Conceptualization, J.A. and R.A.; methodology, J.A.; software, G.A.; validation, J.A., R.A. and G.A.; formal analysis, J.A.; investigation, J.A.; resources, R.A.; data curation, G.A.; writing - original draft preparation, J.A.; writing - review and editing, R.A.; visualization, J.A.; supervision, R.A.; project administration, G.A.; funding acquisition, J.A. All authors have read and agreed to the published version of the manuscript."

Funding: This research received no external funding.

Data Availability Statement: N/A

Conflicts of Interest: The authors declare no conflict of interest.

\section{References}

[1] Collier, B. J., Martin J. B. \& Phyllis G. (2009). Understanding of Textiles. USA: Pearson Publishers.

[2] Design Council (2010). Multi-disciplinary Design Education in the UK, Report and Recommendations from the Multi-Disciplinary Design Network. Available at http://www.designcouncil. org.uk/documents/documents/ ourwork/mdnetwork/mdnetwork_finalreport.pdf (Accessed: $7^{\text {th }}$ March, 2021)

[3] Clarke, S. (20110. Textile Design: Portfolio Series. England: Laurence King Publishing.

[4] African Heritage (2014). Adinkra Symbols and the Rich Akan Culture. Available at https://afrolegends.com. (Accessed: $7^{\text {th }}$ March, 2021).

[5] Adome, D., Appau, A. E., \& Kquofi, S. (2016) Adinkra: An Epitome of Asante Philosophy and History. Journal of Research on Humanities and Social Sciences, 6, 42-53.

[6] Wilson, N. (2018) Bogolanfini. Available at http://bogol anfini.wordpress.com/about/ history-of-malian-mudcloth/ (Accessed: $14^{\text {th }}$ April, 2021)

[7] Friedland, S. \& Pina, L. (1999). African Fabric Design. Pensilvania-USA: Schiffer Publishing Limited.

[8] Luke-Boone, R. (2001), African Fabric (Sewing Contemporary Fashion with Ethnic Flair), USA: Krause Publications.

[9] Polakoff, C. (1982) African Textiles and Dyeing Techniques. London - UK: Routledge and Kegan Paul Ltd.

[10] Ablade, G. (1971), Chart: Adinkra Symbolism. Accra - Ghana: Liberty Press Ltd.

[11] Nakunte, D. (1993). Bogolanfini Patterns, New York - USA: Fashion Institute of Technology. 